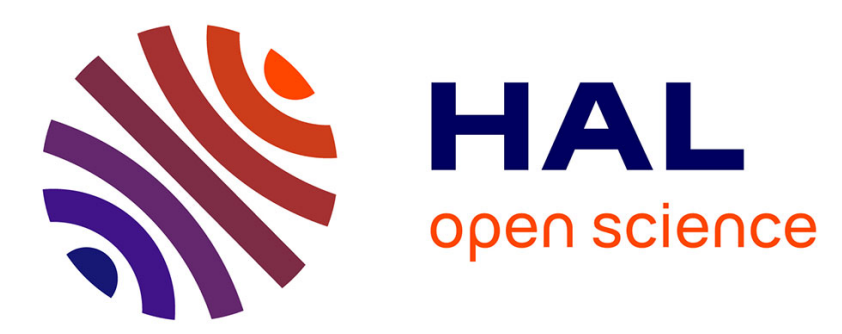

\title{
La Grotte de Roquecourbère (Betchat, Ariège) : ses industries lithiques solutréennes et la révision critique de son art pariétal
}

Pascal Foucher, Cristina San Juan

\section{- To cite this version:}

Pascal Foucher, Cristina San Juan. La Grotte de Roquecourbère (Betchat, Ariège) : ses industries lithiques solutréennes et la révision critique de son art pariétal. Bulletin de la Société préhistorique française, 2000, 97 (2), pp.199-210. hal-00831885

\section{HAL Id: hal-00831885 \\ https://hal.science/hal-00831885}

Submitted on 10 Jun 2013

HAL is a multi-disciplinary open access archive for the deposit and dissemination of scientific research documents, whether they are published or not. The documents may come from teaching and research institutions in France or abroad, or from public or private research centers.
L'archive ouverte pluridisciplinaire HAL, est destinée au dépôt et à la diffusion de documents scientifiques de niveau recherche, publiés ou non, émanant des établissements d'enseignement et de recherche français ou étrangers, des laboratoires publics ou privés. 


\title{
Pascal FOUCHER et Cristina SAN JUAN \\ La Grotte de Roquecourbère (Betchat, Ariège): ses industries lithiques solutréennes et la révision critique de son art pariétal
}

\begin{abstract}
Résumé
Les auteurs présentent une nouvelle étude sur l'industrie solutréenne de la grotte de Roquecourbère, avec quelques éléments de réponse sur l'origine des matières premières, puis la replacent dans le contexte pyrénéen. À cette occasion, une nouvelle analyse des gravures pariétales, découvertes en 1975, est proposée: il existe suffisamment d'arguments pour les considérer comme récentes, certains graffiti pourraient être mis en relation avec des occupations occasionnelles de la grotte pendant la Ire Guerre mondiale.
\end{abstract}

\begin{abstract}
The authors present a new study of the Solutrean industry of Roquecourbère cave, with some results concerning the origin of the lithic raw material, and place it within the Pyrenean context. A new analysis of the parietal engravings, discovered in 1975, is also proposed. The arguments presented in this paper are sufficient to suggest that the engravings are not very ancient; certain graffiti seem to date from occasional occupation of the cave during World War II.
\end{abstract}

\section{LES INDUSTRIES SOLUTRÉENNES DE ROQUECOURBËRE (COLLECTION DU MUSEUM D'HISTOIRE NATURELLE DE TOULOUSE)}

\section{Introduction}

La grotte de Roquecourbère est l'un des rares sites d'habitat solutréens connus dans les Pyrénées centrales, les deux autres étant les abris des Harpons et des Rideaux, dans les gorges de la Save (fig. 1). Malheureusement, il appartient à la longue liste des "sites martyrs" de la Préhistoire pyrénéenne. Découvert (et littéralement vidé) par J. Cazedessus en 1927, il a livré des industries attribuables au Moustérien, au Solutréen et au Magdalénien. Les descriptions publiées par le fouilleur sont très succinctes et ne nous donnent qu'une idée assez vague des différentes occupations préhistoriques, puisqu'il ne fournit pas de détails de la stratigraphie. Les deux articles parus en 1930 et 1953 sont identiques.
Sans que nous connaissions les raisons objectives de son choix, c'est l'industrie solutréenne qui semble avoir retenu l'attention de Cazedessus, si l'on considère le nombre de planches qu'il lui consacre. L'ensemble de la collection J. Cazedessus a été vendu au Field Museum de Chicago. Heureusement, une sélection des plus belles séries d'outils a été conservée par le $\mathrm{Mu}$ séum d'Histoire Naturelle de Toulouse, grâce à l'action déterminante en son temps du Comte $\mathrm{H}$. Bégouën (1935).

Nous avons pu examiner 28 pièces en silex (dont 23 sont typiquement solutréennes) et un fragment de ce qui doit être une sagaie.

Cet ensemble constitue la collection actuellement conservée au Muséum d'Histoire Naturelle de Toulouse $^{1}$ (H. Bégouën évoque une trentaine de pièces dans sa publication de 1935). Tous les objets dessinés par J. Cazedessus, H. Bégouën et P. Laurent ont été retrouvés. Nous fournissons un montage des illustrations anciennement publiées qui en faisait alors le seul panorama connu (fig. 3). Il subsistait une dizaine d'autres pièces non publiées, tout aussi intéressantes et 


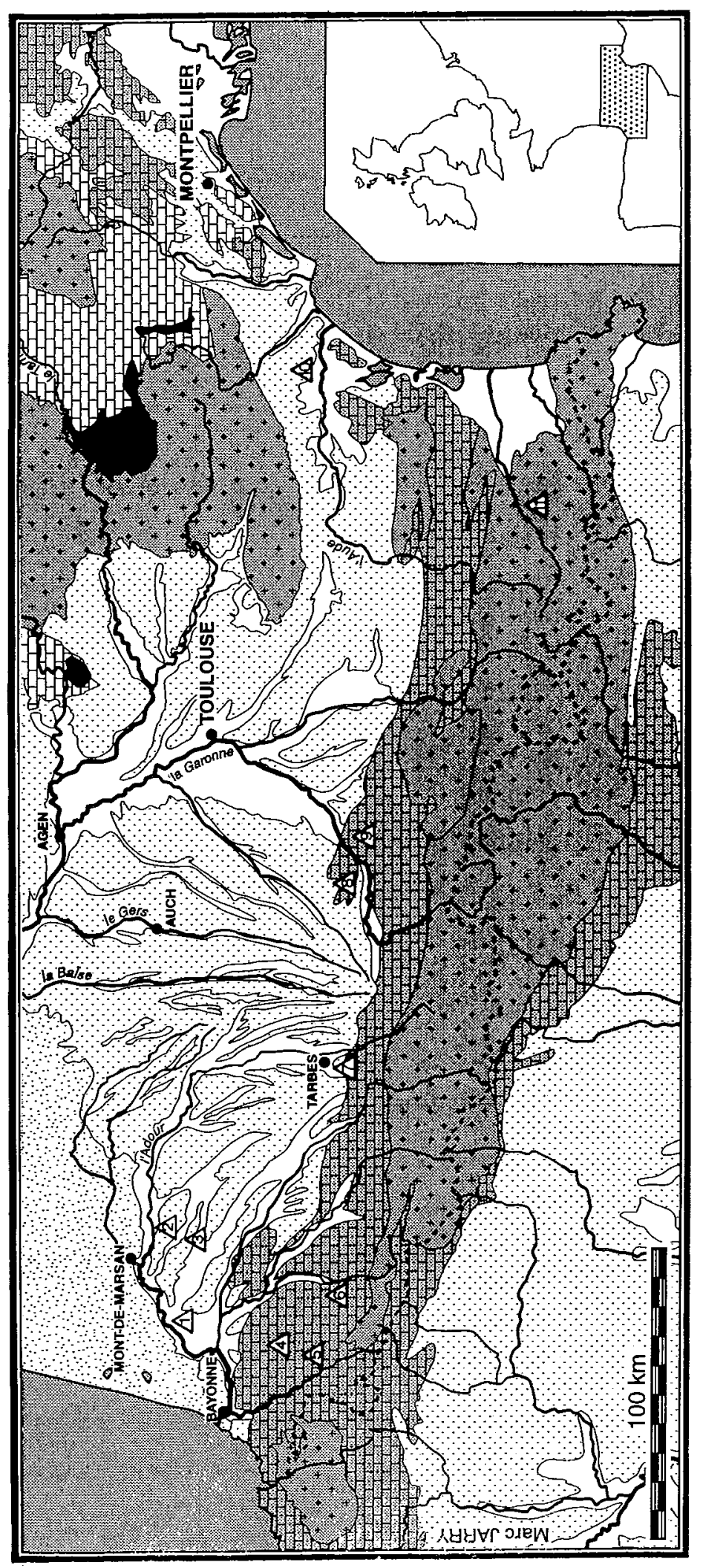

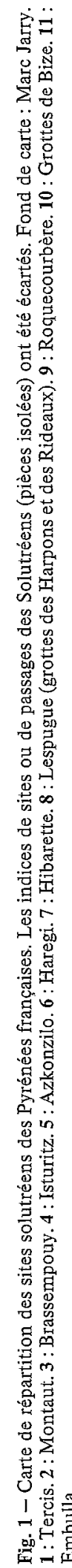

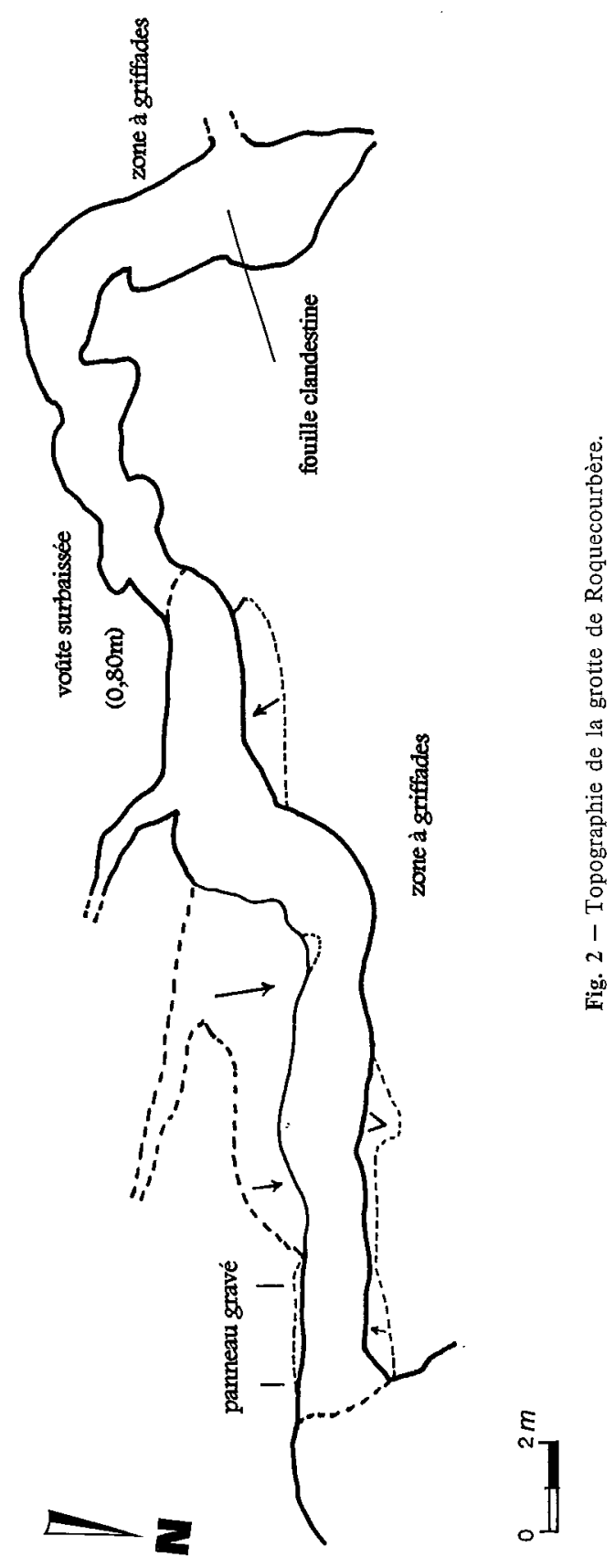



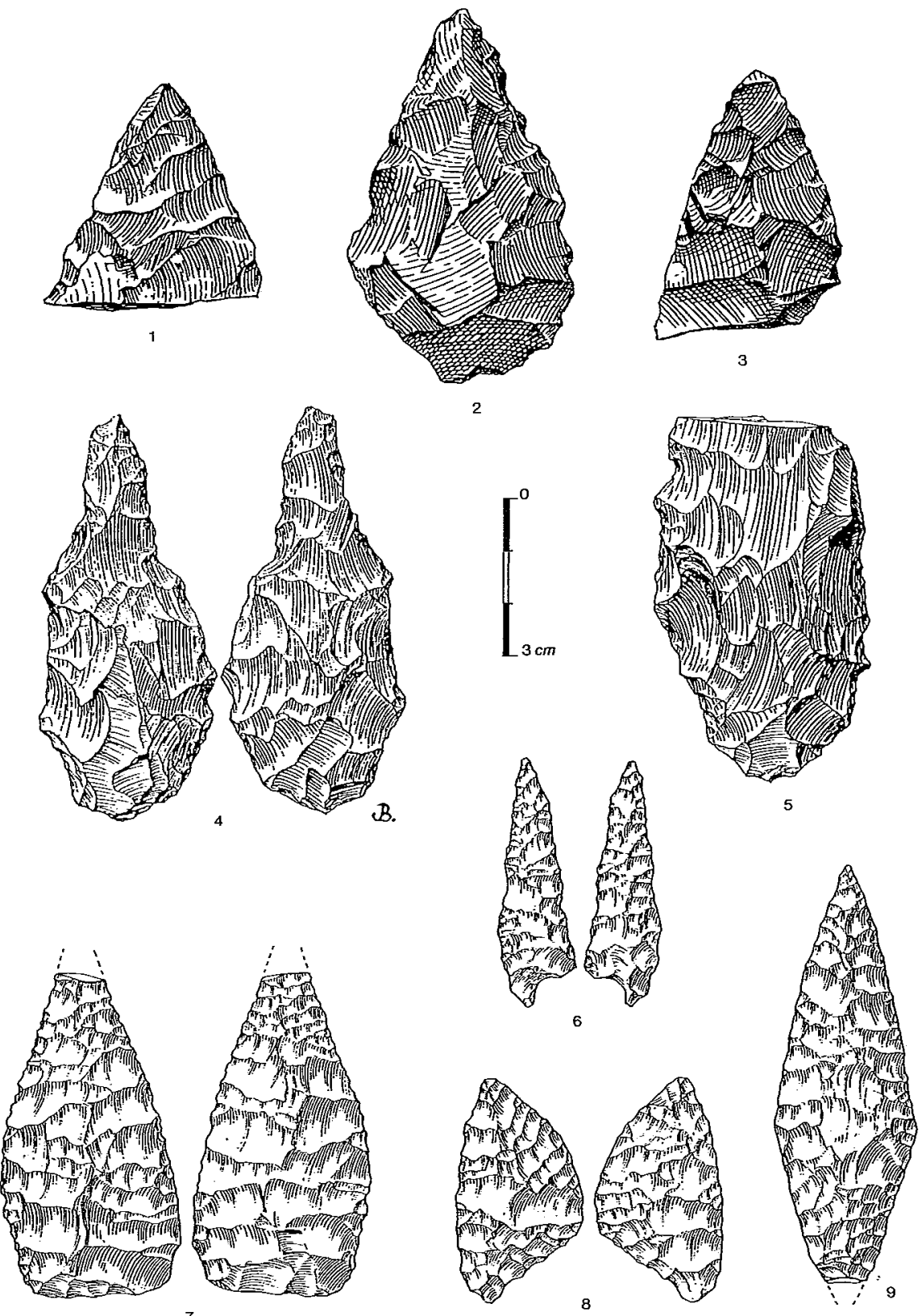

Fig. 3 - Montage de pièces publiées de l'industrie solutréenne de Roquecourbère. 1 à 3: d'après Cazedessus (1930). 4-5: d'après Bégouën (1935). 6 à 9 : d'après Smith (1966). Matière première : 1 à 5 , 7 et 9: "bleu" pyrénéen. $6:$ Montsaunès. 8: Tercis.

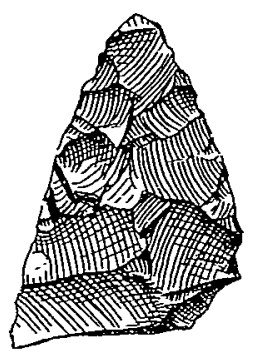

3 inédites que nous avons étudiées et dessinées (fig. 4-5).

\section{Aperçu typologique}

La majorité des pièces solutréennes appartiennent au type "feuille de laurier", et la plupart sont cassées. Elles correspondent souvent à des stades différents de façonnage; cela va de la simple ébauche à peine mise en forme, en passant par la pièce bifaciale de taille sommaire, jusqu'à l'outil finement retouché. Certaines sont à base oblique ou droite (fig. $5, \mathrm{n}^{0 \mathrm{~s}} 2,4$ ), se rapprochant du type "Brassempouy-Isturitz" défini par R. et $S$. de Saint-Périer (1952). Il existe un très bel exemplaire de feuille de laurier asymétrique de type "Montaut (fig. $3, n^{\circ} 8$ ). La pièce $n^{\circ} 5$ (fig. 5), que 1'on pourrait qualifier de feuille de saule à base oblique, est proche de celle du gisement d'Azkonzilo publiée par $\mathrm{Cl}$. Chauchat (1990, fig. $2, n^{0} 2$ ). Bien que l'effectif des pièces solutréennes soit faible, on y retrouve presque tous les types qui ont caractérisé le Solutréen des Landes/Pays basque. 

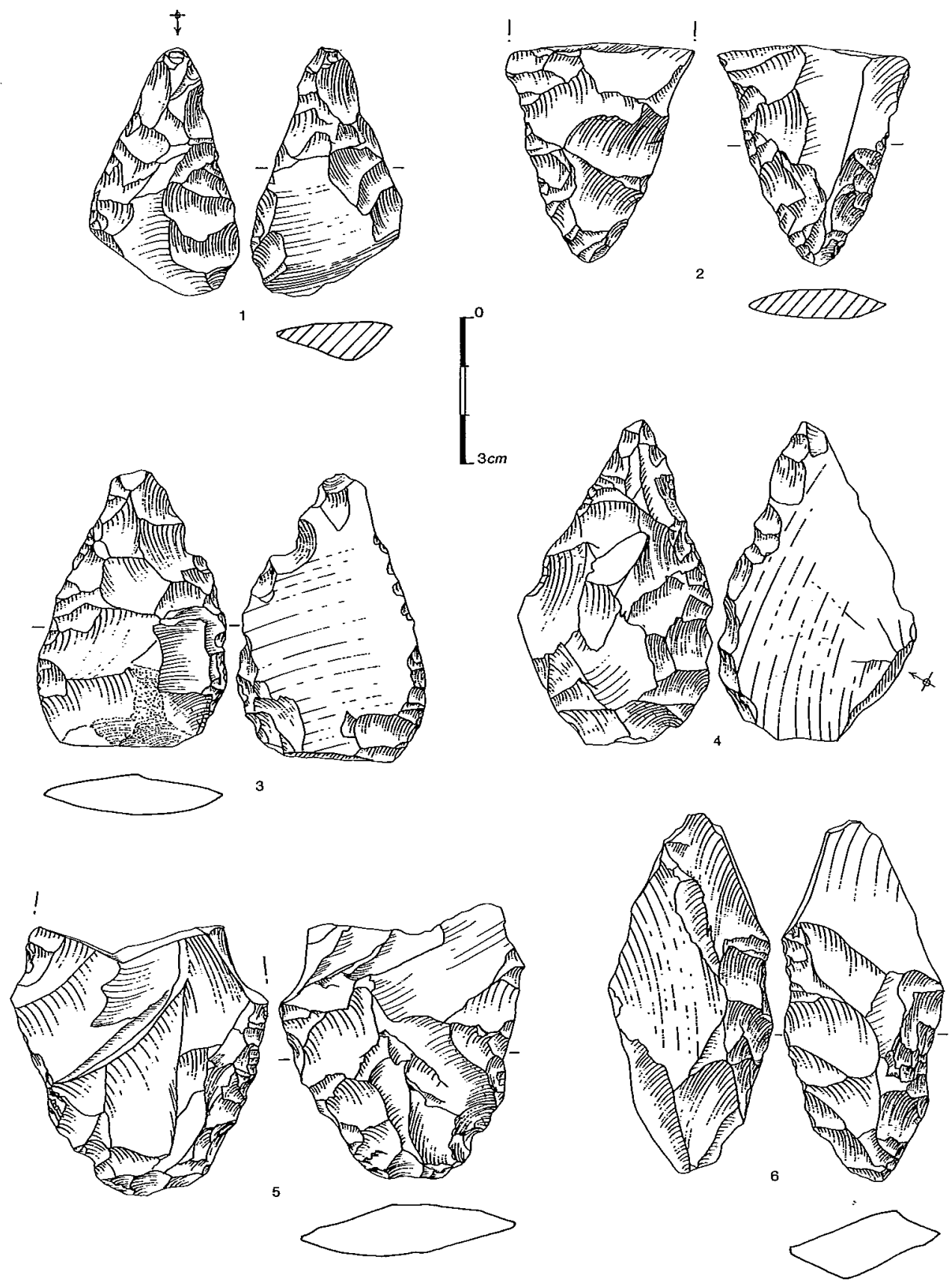

Fig. 4 - Industrie solutréenne de Roquecourbère : ébauches. Matière première: "bleu” pyrénéen ; dessin P. Foucher.

Il faut souligner la présence de pièces exceptionnelles :

- une pointe foliacée à base "encochée" (fig. $3, \mathrm{n}^{0}$ 6). H. Bégouën évoque à son sujet un "pédoncule brisé ". En réexaminant l'objet, il n'y apparaît pas de cassure franche. Il a pu être cassé dans un premier temps puis réparé. Ce qui expliquerait peut-être sa forme peu conventionnelle;

- une feuille de laurier denticulée (fig. $3, \mathbf{n}^{0} 7$ ). H. Bégouën avait déjà noté son caractère quasi unique puisqu'il n'en connaissait qu'un autre exemple trouvé dans les Landes, mais dont la denticulation n'était pas aussi bien réalisée. Sur la feuille de laurier de Roquecourbère, elle se répartit régulièrement sur les deux bords. Le tailleur l'a réalisée à partir d'une seule face et cette opération technique a parachevé le façonnage de la pièce. Il existe une autre pièce qui lui est comparable, celle trouvée dans le solutréen de la grotte d'Embulla dans les Pyrénées orientales (fig. 6) ;

- une pointe foliacée sublosangique courte. C'est une petite pointe, finement retouchée sur les deux faces dont la forme losangique est assez surprenante pour le monde solutréen pyrénéen, bien qu'elle puisse rentrer dans la marge de variations du type de 

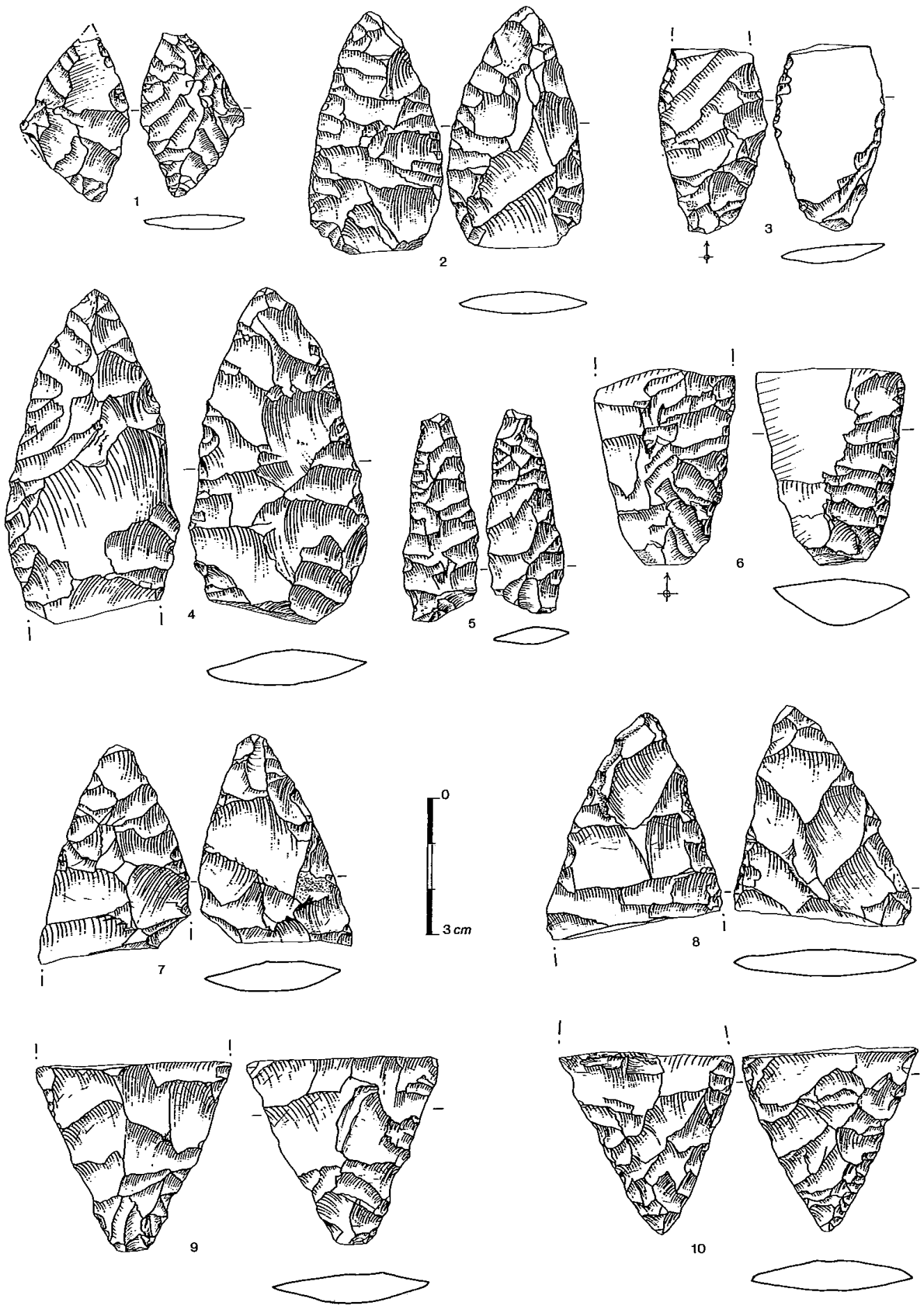

Fig. 5-Industrie solutréenne de Roquecourbère : pièces foliacées. Matière première : $2:$ Montsaunès (ou Flysch). 4 et 9 : Hibarette. Les autres pièces sont élaborées à partir du "bleu" pyrénéen; dessin P. Foucher.

Montaut, mais d'une taille très réduite. Elle n'est pas sans rappeler certaines petites pointes foliacées des sites de l'axe rhodanien (Solutré, Aiguèze).

Ces deux dernières pièces se singularisent, tant par le procédé technique employé (pour la première), que par la forme (pour la seconde), qui ne seront développés qu'à partir du Chalcolithique. Le manque de rigueur de la fouille de J. Cazedessus pourrait inciter à croire à la possibilité de mélanges avec une occupation beaucoup plus récente que le Solutréen. Néanmoins, le support foliacé à la denticulation est de facture solutréenne, et la patine de la pointe losangique est identique à celle des autres pièces solutréennes, si bien que nous sommes convaincus de leur attribution à ce groupe culturel. 

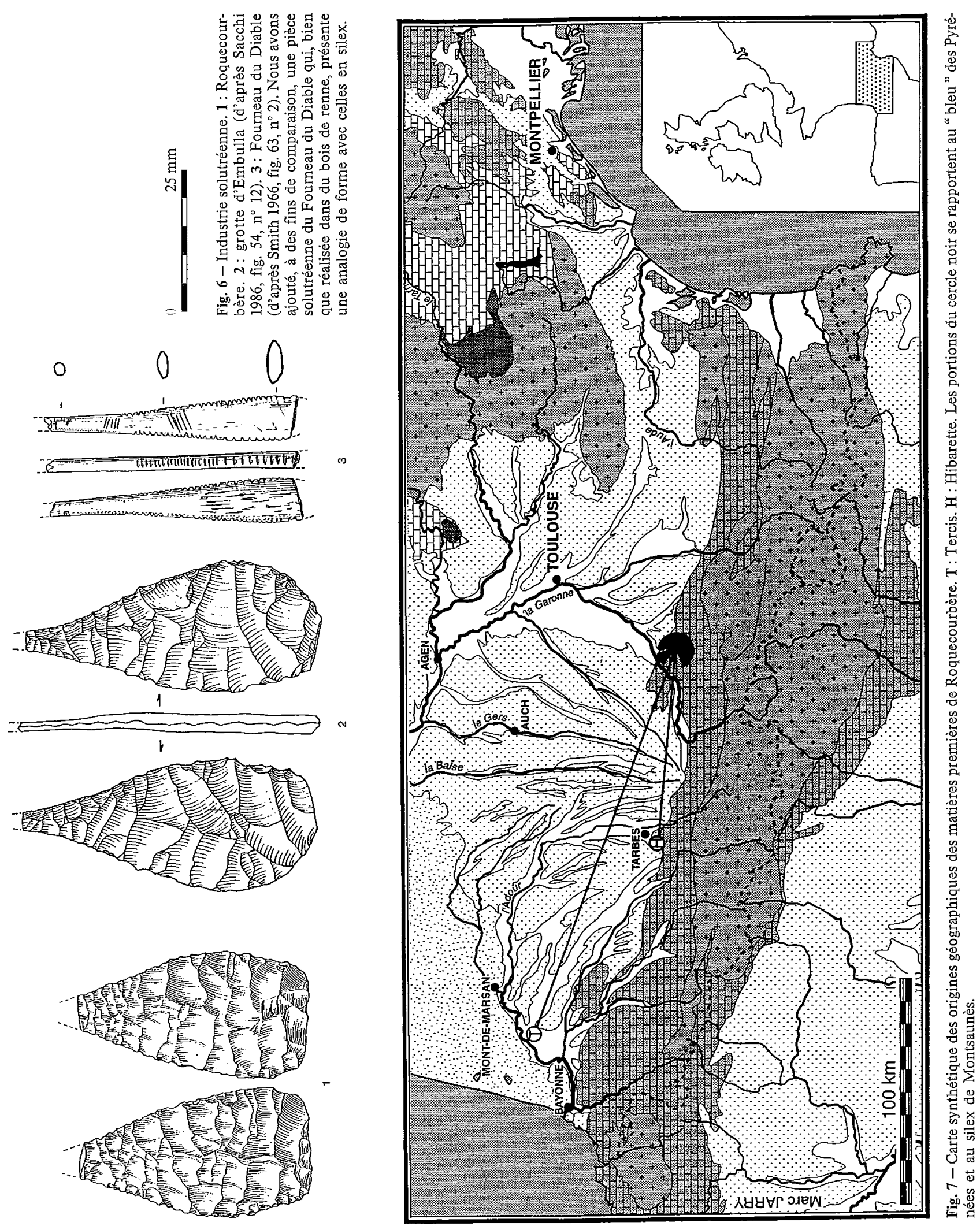


\section{Relations entre la typologie et la matière première ${ }^{2}$}

Dans les différents types présents - pointe foliacée à base concave, feuille de laurier losangique, feuille de laurier asymétrique de type "Montaut" (fig. $3, n^{\text {os }} 6,8$, $9)-$ H. Bégouën puis $\mathrm{Ph}$. Smith avaient déjà reconnu une nette influence des groupes solutréens des Landes, du Pays basque, voire des Cantabres. Il nous a semblé intéressant de vérifier si cette influence était aussi perceptible dans le choix des matières premières employées.

Le nombre de pièces étant peu important (23), nous présentons nos résultats en forme de tableau simple ( $c f$. tab. I).

\begin{tabular}{|c|c|c|c|c|}
\hline Petites Pyrénées & Montsaunès & Hibarette & Tercis & Total \\
\hline 18 & 2 & 2 & 1 & 23 \\
\hline
\end{tabular}

Tab. I - Répartition par type de matière première des outils solutréens.

La presque totalité des outils solutréens (18) a été réalisée dans du silex "bleu" des Pyrénées. La pointe losangique (fig. $3, \mathrm{n}^{\circ}$ 9) dont Smith avait noté la parenté typologique avec le type losangique cantabrique de Jordá Cerdá (1966, p. 336) a été taillée dans ce silex calcédonieux qui présente exactement les mêmes caractéristiques de texture et de patine que celui du gite de Belloc, situé à quelques centaines de mètres de la grotte.

Deux supports en silex du type de "Montsaunès" proviennent de gîtes situés à une dizaine de kilomètres à peine.

Il existe trois pièces façonnées dans un silex d'origine éloignée : une feuille de laurier entière et le fragment d'une autre proviennent d'un gîte à silex de type "Hibarette" (125 km) ; la belle pièce foliacée asymétrique du type de Montaut (fig. $3, \mathrm{n}^{\circ} 8$ ) a été élaborée sur un support provenant des gîtes de Tercis dans les Landes $(192 \mathrm{~km})$. Ces deux dernières provenances confortent cette influence occidentale.

J. Cazedessus avait noté dans sa publication qu'il avait ramassé " des percuteurs, des nucléus volumineux, des grands éclats..." (1930, p. 468) et H. Bégouën déplorait de ne pas avoir pu récupérer lors de la vente des collections au Musée de Chicago “ces innombrables éclats, rappelant les écailles de poissons" (1935, p. 131). Ces éléments décrits, plus la présence d'ébauches de pièces bifaciales dans la collection étudiée, évoquent bien une activité de taille intensive dans les niveaux solutréens de la grotte. Par ailleurs, comme nous l'avons évoqué plus haut, la presque totalité des produits finis que nous avons pu réexaminer a été taillée à partir de silex local : la matière première a été recherchée à proximité du gisement, dans les gîtes qui se trouvent sur le rebord du plateau où s'ouvre la cavité.
Il faut mentionner ici une découverte que nous avons pu faire à la suite de l'examen de l'industrie des fouilles Russell/Begouën, menées en 1931 dans le vallon que domine la grotte de Roquecourbère (Bégouën, Russell, 1933). Ces deux préhistoriens ont pu mettre en évidence un atelier de taille qu'ils avaient alors attribué à un "Aurignacien inférieur : Aurignacien I de Breuil, faciès de 1'abri Audi". Dans la collection qui est aujourd'hui conservée au Muséum, il existe quelques pièces très caractéristiques, comme des éclats de façonnage de pièces foliacées, qui évoquent plutôt une technologie solutréenne (Foucher, San Juan, en préparation). Cette redécouverte d'un atelier de taille de plein air solutréen relève l'importance du site de Roquecourbère dans le monde solutréen des Pyrénées, en particulier dans ses dimensions paléo-ethnologiques; il apparaît comme un site complexe, alliant vraisemblablement un habitat en grotte avec des ateliers de plein air à proximité.

\section{Conclusion}

Bien que le Solutréen pyrénéen soit relativement méconnu en raison du faible nombre de sites, de l'absence de stratigraphies bien développées, et de la mauvaise qualité des fouilles anciennes qui, malheureusement, ont sacrifié des sites qui auraient pu être décisifs pour la connaissance de cette culture, les maigres informations auxquelles on peut faire référence montrent à l'évidence un courant culturel qui longe la chaîne pyrénéenne d'une mer à l'autre.

Les pièces foliacées de Roquecourbère sont caractérisques des ensembles solutréens de la zone des Pyrénées occidentales (Landes comprises). L'étude des matériaux siliceux confirme bien une ouverture sur les territoires de l'Ouest pyrénéen (fig. 7) : les deux feuilles de laurier en silex d'Fibarette et la pointe de Montaut en silex de Tercis en constituent une preuve matérielle. Le gîte de matière première d'Hibarette, certainement l'un des plus importants dans les Pyrénées centrales, apparaît comme une station intermédiaire dans les déplacements des groupes solutréens, qui semblent avoir suivi une route parallèle aux Pyrénées. Le fait de retrouver des pièces d'influence typologique occidentale, taillées dans du silex issu de gîtes proches de la grotte de Roquecourbère, accompagnant des pièces réalisées sur des supports provenant des Landes et de la Bigorre, pourrait suggérer une orientation des déplacements dans le sens ouest est. Ces derniers ont dû se prolonger jusqu'aux Pyrénées orientales: la grotte d'Embulla pourrait être un des jalons les plus orientaux de ce groupe pyrénéen qui se placerait plutôt dans la phase supéricure de la chronologie du monde solutréen. Les études que nous projetons de mener dans les années à venir (Foucher, San Juan, 1998) s'organiseront autour de deux axes : le premier consistera à trouver de nouveaux sites en stratigraphie dans les Pyrénées centrales; le second, compte tenu de l'information disponible, prétend étudier les liens ou les relations éventuelles qui unissent le groupe pyrénéen avec les Cantabres, et les bordures méditerranéennes, entre la vallée du Rhône et le Levant espagnol. 


\section{RÉVISION DE L'ART PARIÉTAL DE LA GROTTE DE ROQUECOURBÈRE}

\section{Rappel de la problématique}

En 1975, R. Simonnet découvrit un petit ensemble de gravures sur un panneau situé juste à l'entrée de la grotte, sur la paroi gauche. Parmi une série de traits et d'inscriptions gravés, il y reconnut un bouquetin. Le problème de l'authenticité des gravures se posa d'emblée en raison de leur situation proche de l'entrée, de l'aspect fruste du bouquetin et de son caractère isolé. En 1984, J. Clottes notait: "[...] Le dos de l'animal est constitué par une fissure naturelle de la roche. Sur la même paroi, plusieurs groupes différents de traits paraissent patinés ou relativement anciens. Néanmoins, des noms et des dates ont été gravés plus ou moins récemment dans leur voisinage et certaines de ces inscriptions sont tout aussi patinées que les traits ou que la gravure du bouquetin. Cela s'explique par la proximité de l'entrée, par les ruissellements, les algues et les mousses qui affectent la roche et provoquent le vieillissement rapide de cette gravure. L'utilisation d'un contour naturel, comme le choix du sujet, pourraient être des arguments en faveur de l'authenticité. Si cette gravure était authentique, les traces de remplissage visibles sur la paroi indiquent qu'elle était entièrement recouverte par certaines couches archéologiques: elle aurait donc été dégagée par les fouilles de J. Cazedessus, qui ne l'aurait pas vue." (in: L'Art des Cavernes, p. 363).

Les remarques de J. Clottes restent toujours pertinentes. Toutefois, $\mathrm{il}$ faut replacer cette découverte dans le contexte de l'époque: nous étions encore bien loin des découvertes pariétales du Placard, de Cosquer et de Foz Coa, et de leurs conséquences épistémologiques (la première étant la reconnaissance d'un art solutréen bien développé dans la zone franco-ibérique); ce qui explique que cette découverte soit retombée à l'époque dans une certaine indifférence.

En plus de vingt ans, l'état général de conservation du panneau ne semblait pas avoir évolué, si ce n'est en se couvrant davantage de mousse; on ne remarquait pas de graffiti supplémentaires. En revanche, les cornes du bouquetin semblaient avoir été " repassées", leur donnant une coloration plus "fraîche" qu'elles n'avaient pas lors de la découverte. La grotte est depuis toujours ouverte, sans aucune protection, et de nombreuses fouilles clandestines (ou désobstructions) sont à regretter.

Comme nous venons de l'évoquer précédemment, un doute subsistait toujours quant à l'attribution chronologique des gravures, même si une attribution paléolithique pour le bouquetin restait envisageable; des données d'ordre technologique et stylistique plaidaient en ce sens : les tracés multiples des lignes des pattes et des cornes, la représentation en $U$ renversé des pattes. Par ailleurs, on soupçonnait d'autres gravures sous les mousses qui, si elles étaient enlevées, pouvaient éventuellement révéler d'autres motifs et des représentations paléolithiques ou modernes.
Nous avons donc procédé, avec la collaboration de Monique Drieux du laboratoire de restauration Archéologies $^{3}$, au nettoyage du panneau gravé en prenant le maximum de précautions quant à l'intégrité des surfaces de la paroi (Drieux, 1998).

Cette opération s'est déroulée en quatre phases:

- deux demi-journées ont été consacrées à l'aspersion d'un produit algicide ${ }^{4}$ sur le panneau;

- une journée entière, au nettoyage mécanique proprement dit de la paroi : nettoyage à sec avec une petite brosse souple, et humide par tamponnage sur des zones-test autour et à proximité des gravures; des relevés photographiques des gravures ont été réalisés simultanément;

- une autre demi-journée, pour constater l'évolution du nettoyage 5 mois après, et effectuer d'autres observations avec la participation de Robert Simonnet, inventeur des gravures;

- enfin, une dernière journée, destinée à la mise au point des relevés par des observations complémentaires.

Au cours de cette opération, nous en avons profité pour effectuer une fouille soigneuse des fissures du panneau à la recherche d'objets ou de vestiges qui auraient pu être fichés dans la paroi : les résultats ont été négatifs.

\section{Diagnostic sur l'authenticité paléolithique des gravures}

Le panneau gravé se situe à l'entrée de la galerie, sur la paroi gauche, en pleine lumière du jour (fig. 2). Sa partie supérieure constitue l'unique tronçon des parois de la grotte qui présente un calcaire sain, non altéré. Les surfaces du reste du réseau sont de type mondmilch, ou présentent une texture crayeuse, très tendre, sur laquelle on peut noter en plusieurs endroits de nombreuses griffades d'animàux.

Étant donné les différents vestiges du niveau de remplissage qui subsistent sur les parois, il semble que les gravures existantes n'aient pas été recouvertes par les sédiments archéologiques. L'inventeur et le fouilleur de la grotte, J. Cazedessus, n'a donné que très peu d'indications sur les conditions de la découverte du gisement et la stratigraphie du remplissage: "Les travaux [...] révélèrent dans ce que nous croyions être un autre abri, une galerie tortueuse d'une largeur et d'une hauteur moyenne de 2 mètres. Sa profondeur demeure inconnue, car nous n'avons enlevé en longueur que 50 mètres du remplissage. Celui-ci, presque partout, atteignait le plafond. La terre des 30 premiers mètres, quoique compacte, fut enlevée facilement à la brouette [...]. Dès le surplomb, 3 foyers superposés apparurent..." (1930, p. 467-468). Malgré sa remarque qui semble exagérée (un remplissage qui atteindrait le plafond sur $50 \mathrm{~m}$ alors que le développement total de la cavité n'excède pas 30-35 m), il semblerait plus envisageable que l'entrée de la grotte ait été colmatée jusqu'à la voûte par un bouchon de sédiments en forme de dôme qui ne devait 
pas excéder quelques mètres de long; puis, il devait se continuer en présentant un pendage oblique, laissant libre une bonne partie supérieure des parois. Il existe vraisemblablement une relation de cause à effet entre les zones recouvertes et altérées d'une part (partie inférieure du panneau), et d'autre part celles découvertes et non altérées (partie supérieure = zone gravée). L'organisation des unités graphiques du panneau (fig. 8-9) est simple : le bouquetin occupe une zone centrale autour de laquelle se distribuent à droite deux

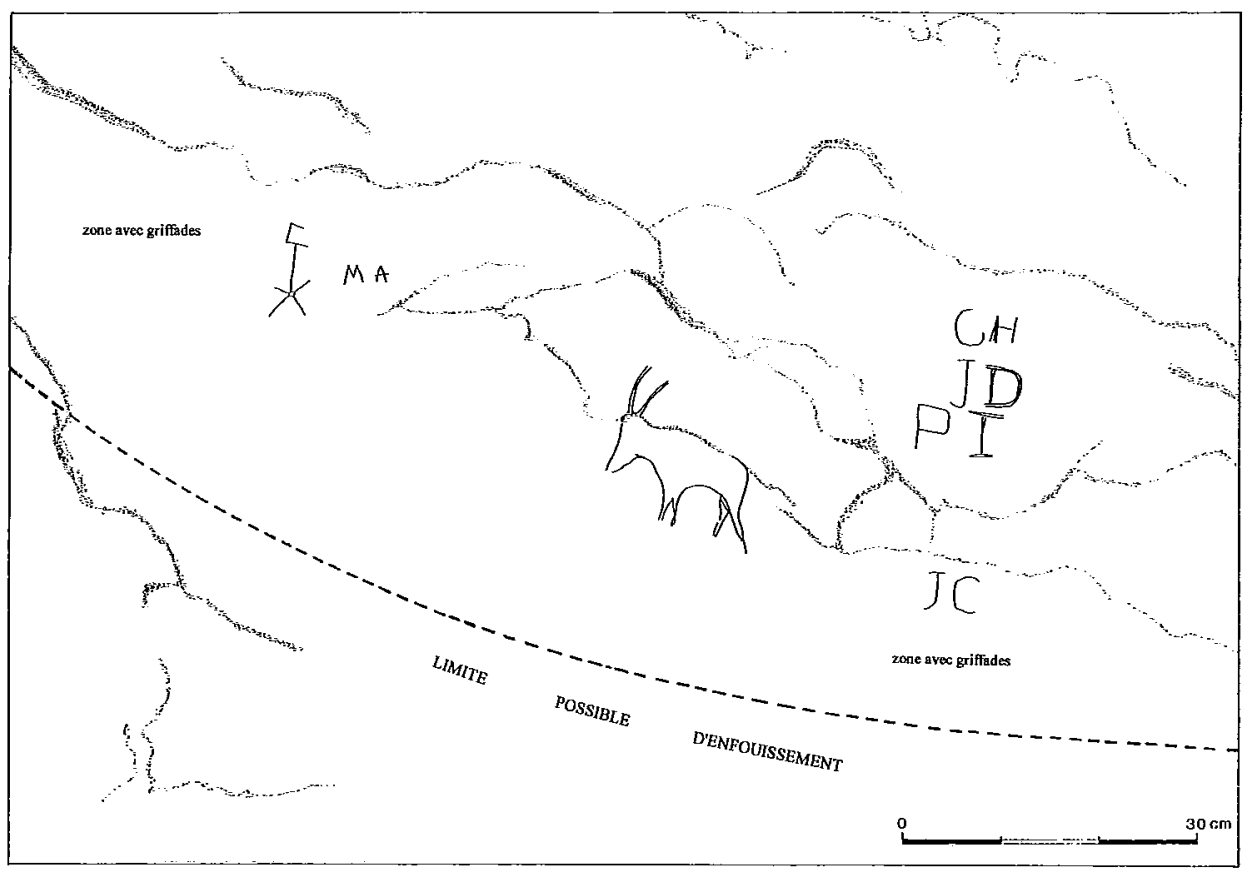

Fig. 8a (ci-contre) - Relevé du panneau gravé

Fig. 8b (ci-dessous) - Vue générale du panneau gravé.

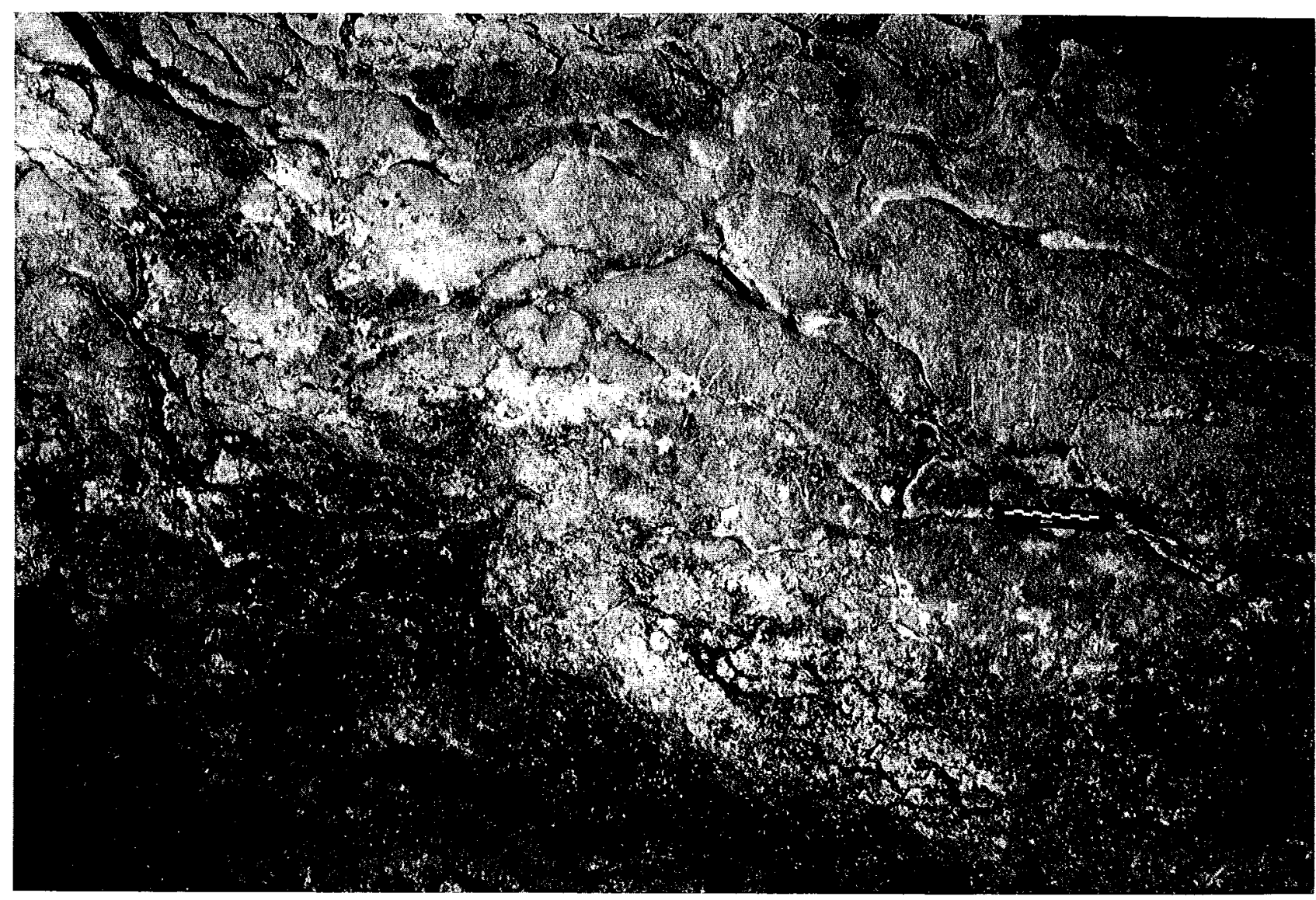



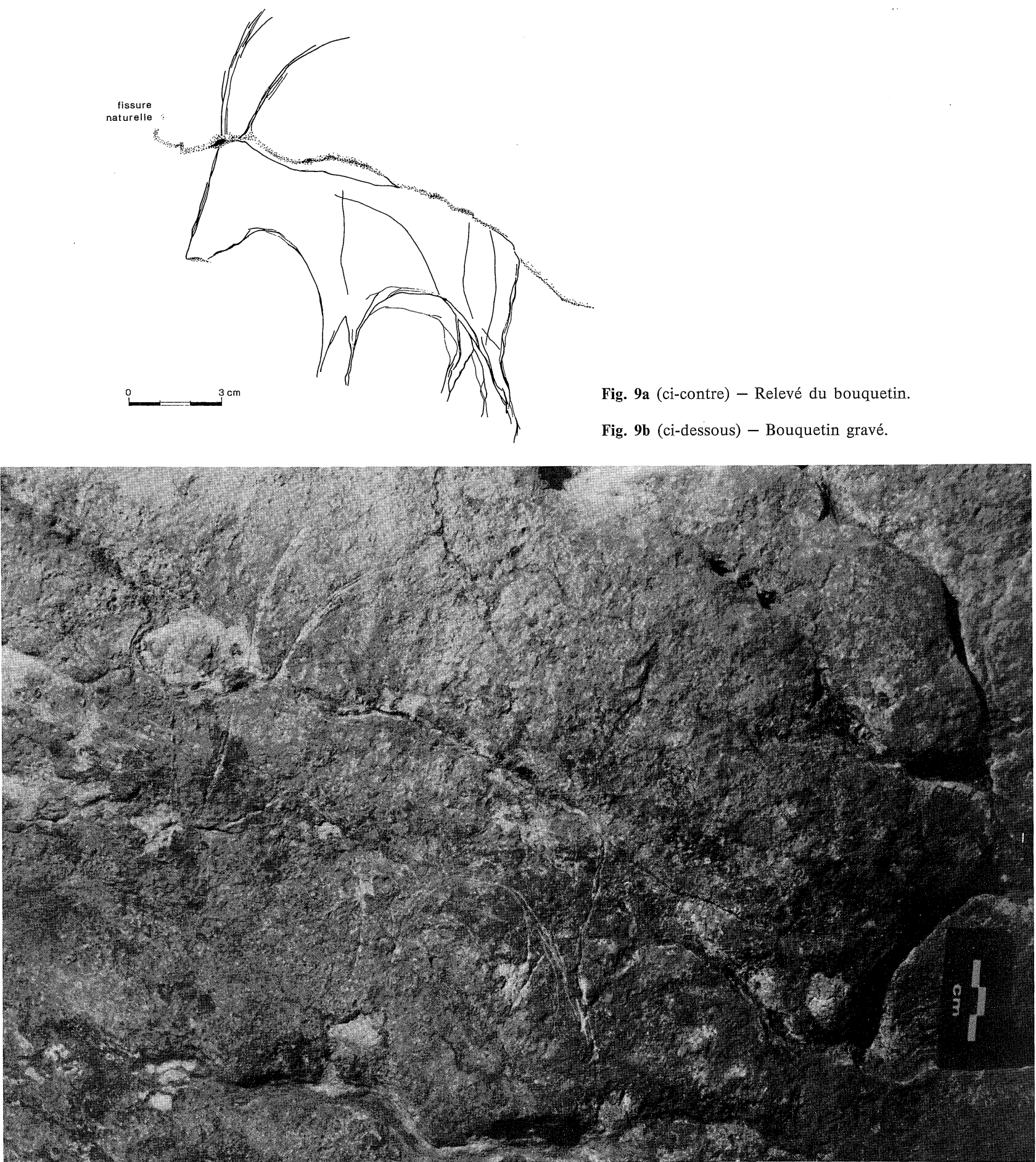

plages de graffiti et à gauche deux autres plages présentant une rouelle et des griffades d'animaux. Le terme de "plage" définit une surface irrégulière sans aspérité, délimitée par des fissures naturelles de la roche.

Le bouquetin est de petite taille : $15,4 \mathrm{~cm}$ du museau à la croupe (fig. 9). Sa silhouette intègre des fissures naturelles pour la représentation du dos et de la partie terminale du museau. Les cornes sont vues en perspective tordue ainsi que les pattes antérieures. Le train arrière respecte les canons de la perspective normale. Des motifs géométriques viennent se placer à l'intérieur du corps de l'animal et au-dessous de son museau.

Considérant les différentes techniques de représentation employées (différentes perspectives, intégration du support, style, etc.), ce bouquetin présente une facture paléolithique qui le rapprocherait, par analogie, de 
certains bouquetins rencontrés dans les grottes de l'Ardèche, souvent d'attribution solutréenne. Par ailleurs, l'étude des différents types de gravures renforce encore cette proposition; on distingue par exemple un tracé de type "code barre" (Fritz, 1995) sur les cornes, ou encore des tracés multiples, discontinus, de section en V dissymétrique pour le dessin du ventre, qui dénotent une possible utilisation d'un outil en silex.

Cependant, l'analyse des sillons gravés laisse planer de sérieux doutes quant à un âge paléolithique de la représentation. En effet, aucun trait n'est patiné. On peut constater qu'il existe trois états de surface pour les traits gravés : le fond de certaines gravures apparaissent d'un blanc éclatant, correspondant à la roche saine mise à nue, pour d'autres c'est une coloration brunmarron qui domine. Cette dernière ne peut être considérée comme une patine. En effet, il arrive souvent qu'une même gravure présente en continu ces deux états de surface : ce fait traduit l'intensité différentielle de la pression que le graveur a exercée sur la paroi avec son instrument; avec une pression forte, il a atteint la roche saine ; avec une pression moindre, le graveur n'a seulement imprimé que la partie superficielle de la paroi qui possède cette coloration brun-marron. I1 existe un troisième état intermédiaire que l'on trouve sur les traits du ventre qui prennent une coloration grisblanc, d'un aspect très frais. Enfin, il existe en plusieurs points de la représentation (haut du museau, cou, antérieur droit, postérieurs gauche et droit) des petites zones d'écaillement de la paroi qui ont dû se produire au moment des premiers coups de gravure, faisant ainsi apparaître la roche saine; dans un deuxième temps, l'opérateur y est revenu, surgravant le fond de ces écailles.

La confrontation avec les caractéristiques des sillons des graffiti modernes qui entourent le bouquetin efface les doutes qui pouvaient subsister puisqu'on retrouve exactement les mêmes gradations de texture et de coloration. Ainsi, la réalisation de cette représentation ne peut être que moderne. Il se pourrait même qu'un des signataires des graffiti en soit l'auteur puisque, paradoxalement, alors que cette partie de la paroi est couverte d'initiales, aucune inscription ne vient surcharger le bouquetin!

Depuis un peu moins de 25 ans, l'âge de l'art pariétal de 1a grotte de Roquecourbère demeurait incertain. La modeste opération de nettoyage du panneau a permis d'obtenir une meilleure lisibilité des gravures, et surtout des graffiti modernes ; il nous a été possible d'établir une parenté technique, doublée d'une identité des états de surface des gravures, entre la réalisation du bouquetin et les inscriptions récentes. On doit désormais considérer la représentation du bouquetin comme moderne, certes très intelligemment réalisée pour un "faux" puisqu'elle intègre les techniques de représentation paléolithiques, mais néanmoins sans grande grâce stylistique.

\section{NOTES}

(1) Nous tenons à remercier M. Duranthon et Mme Lasbails du Museum d'Histoire Naturelle de Toulousc pour leur aimable accueil. (2) La détermination des matières premières a été faite avec la collaboration de R. Simonnet, M. Allard et S. Lacombe. Nous avons intégré la problématique que R. Simonnet avait déjà longuement précisée (1998, 1996, 1985, 1982). Dans la terminologie employée, le choix des termes est dicté par des considérations d'ordre géographique; de même nous utilisons à dessein l'usage d'appellations comme "Montsaunc̀s", "Tercis", "Ilibarette", etc. qui définissent des gîtes éponymes đe matière première, c'est-à-dire qu'il existe sullissamment de caractères discriminants pour les silex recontrés dans Ies affleurements géologiques de ces communes pour en faire un type. Le contexte géomorphologique des Pyrénćcs ćtant ce qu’il est, il n’y a guc̀rc de possibilités de trouver des silex avec les mêmes caractéristiques dans de nouveaux gîtes très éloignés des gîtes éponymes (sauf en ce qui concerne le "bleu" pyrénéen qui se retrouve tout au long des Petites Pyrénées). En conséquence, pour ces silex typiques, leur origine géomorphologique coïncíde avec une origine géographique prócise. Ainsi, l'expression "silex de Montsaunès " doit être comprise dans le sens de : silex de type "Montsaunès".

(3) Archéologies, Laboratoire de conservation ef de restauration, 5 passage de Véronne, 31500 Toulouse.

(4) Alkgl dinéthyl benzyl ammonium chlorure.

\section{RÉFÉRENCES BIBLIOGRAPHIQUES}

ALLARD M. (1989) - Collection R. et S. de Saint-Périer à Lespugute (Haute-Garonne), Préhistoire ariégeoise, Bulletin de la Société préhistorique Aniège-Pyrénées, XLIV, p. 203-224, 9 fig.

ALLARD M., JARRY M. (1993) - Collection R. et S. de Saint-Périer à Saint-Gaudens (Haute-Garonne), Préhistoire ariégeoise, Bulletin de la Société préhistorique Ariège-Pyrénées, XLVII, p. $47-83,20$ fig.

BEGOUËN H. (1935) - Le Solutréen dans les Pyrénées, Revue anthropologique, $\mathrm{n}^{\circ} 4-6$, p. 126-136, 8 fig.

BEGOUËN H., RUSSELL T. (1933) - La campagne de fouilles de 1931 à Marsoulas, Tarté et Roquecourbère, Toulouse, Privat Éd.

BUISSON D. (1996) - Brassempouy: présentation du site et problèmes posés par les fouilles récentes, in: Delporte $\mathrm{H}$. et Clottes J. (dir.), Pyrénées Préhistoriques - Arts et Sociétés, actes du $118^{e}$ congrès national des sociétés historiques et scientifiques, Pau, 1993, Éditions du C.T.H.S., p. 423-437, 4 fig.

CAZEDESSUS J. (1930) - La galerie de Roquecourbère (Ariège), in : Congrès A.F.A.S., Le Havre.
CAZEDESSUS J. (1953) - La galerie de Roquecourbère (Ariège) Revue de Comminges, $4^{\mathrm{e}}$ trim., p. 175-181, 3 pl.

CHAUCHAT Cl. (1990) - Le Solutréen en Pays basque, in: Kozlowski J. K. (dir.), Feuilles de pierre: les industries à pointes foliacées du Paléolithique supérieur européen, actes du colloque de Cracovie 1989 (VIII $^{e}$ commission de l'U.I.S.P.P.), p. 363-376, 5 fig. (ERAUL, 42).

CLOTTES J. (1976) - Les civilisations du Paléolithique supérieur dans les Pyrénées, in : Lumley $\mathrm{H}$. de (dir.), La Préhistoire française, vol. 1, Paris, C.N.R.S., p. 1212-1231.

DELPORTE H. (1996) - Brassempouy : histoire d'un gisement, in : Delporte H. et Clottes J. (dir.), Pyrénées Préhistoriques - Arts et Sociétés, actes du $118^{e}$ congrès national des sociétés historiques et scientifiques, Pau, 1993, Éditions du C.T.H.S., p. 415-421, 2 fig.

DRIEUX M. (1998) - Opération de nettoyage de la paroi gravée de la grotte de Roquecourbère, in: Foucher P. et San Juan C. (dir), Le complexe gravettien/solutréen des Pyrénées centrales, rapport de 
prospection thématique, Service régional de l'Archéologie de Midi-Pyrénées, p. 22-23.

FOUCHER P., SAN JUAN C. (1998) - Le complexe gravettien/solutréen des Pyrénées centrales: prospection thématique, Bilan scientifique 1997 du Service Régional de l'Archéologie de MidiPyrénées, Ministère de la Culture et de la Communication, p. 232 .

FOUCHER P., SAN JUAN C. (1998) - Le complexe gravettien/solutréen des Pyrénées centrales: rapport de prospection thématique, Service Régional de l'Archéologie de Midi-Pyrénées, 90 p., 38 fig., IV pl.

KOZLOWSKI J.-K., OTTE M. (1990) - Conclusions et perspectives, in: Kozlowski J.-K. (dir.), Fenilles de pierre: les industries d̀ pointes foliacées du Puléolithique supérieur européen, actes du colloque de Cracovie 1989 (VIII" commission de l'U.I.S.P.P.), p. 539-549, 3 fig. (ERAUL, 42).

LACOMBE S. (1998) - Stratégies d'approvisionnement en silex au Tardiglaciaire. L'exemple des Pyrénées centrales françaises Préhistoire ariégeoise, Bulletin de la Société préhistorique AriègePyrénées, t. LIII, p. 223-266, 4 fig.

MEROC L. (1947) - Le silex dans le bassin sous-pyrénéen de la Garonne et son emploi par l'homme préhistorique, Bulletin de la Société archéologique du Midi de la France, $3^{\mathrm{c}}$ série, tome V, p. 234-250.

MEROC L (1953) - La conquête des Pyrćnées par l'Homme, Premier Congrès International de Spéléologie, Paris, t. IV, section 5 , p. 33-51.

PASSEMARD E. (1944) - La caverne d'Isturitz en Pays Basque, Préhistoire, t. IX, p. 7-84, 63 fig., LXIV pl. h.t.

SACCHI D. (1986) - Le Paléolithique supérieur du Languedoc occidental et du Roussillon, Paris, Éditions du C.N.R.S., 284 p., 199 fig., XVI pl. h.l. (XXI ${ }^{\mathrm{e}}$ supplément à Gallia Préhistoire).

S^CCHI D. (1990) - Le Solutréen des Pyrénées méditerranéennes françaises et de leurs abords, in : Kozlowski J.-K. (dir.), Feuilles de pierre: les industries à paintes foliacées du Paléolithique supérieur européen, actes du colloque de Cracovie 1989 (VIII $^{\mathrm{e}}$ commission de l'U.I.S.P.P.), p. 377-392, 9 fig. (ERAUL, 42).

SAINT-PÉRIER R. de (1920) - La grotte des Harpons à Lespugue (Haute-Garonne), L'Anthropologie, 30, p. 209-234.

SAINT-PÉRIER R, de (1921) - Les grottes préhistoriques de Lespugue et de Montmaurin (Haute-Garonne), Revue de Comminges, $2^{\mathrm{e}}$ trim.

SAINT-PÉRIER R. de (1924) - Les fouilles de 1923 dans la grotte des Rideaux à Lespugue (Haute-Garonne), L'Anthropologie, 36 , p. $15-40$.
SAINT-PÉRIER R. de (1926) - Quelques pièces inédites de la grotte des Harpons à Lespugue (Haute-Garonne), L'Homme préhistorique, 7-8.

SAINT-PÉRIER R. de (1952) - La grotte d'Isturitz : les Solutréens, les Aurignaciens et les Moustériens, Paris, Masson, 264 p., 135 fig., XI pl. h.t. (Archives de l'Institut de Paléontologie humaine: mémoire $\mathrm{n}^{\circ} 25$

SIMONNET R. (1976) - Les gisements préhistoriques des gorges de 1a Save dans le massif de Lespugue-Montmaurin, in: IX ${ }^{c}$ Congrès U.I.S.P.P., Nice, Livret-guide excursion A5, p. $117-122$.

SIMONNET R. (1982a) - Grandes lames de silex dans le Paléolithique supérieur des Pyrénées centrales, Préhistoire ariégeoise Bulletin de la Société préhistorique Ariège-Pyrénées, t. 37 p. 61-106, 12 fig., 1 tabl.

SIMONNET R. (1982b) - Carte des gîtes à silex des Pré-Pyrénées, in : La Préhistoire en Quercy dans le contexte de Midi-Pyrénées, Congrès Préhistorique de France, Montauban-Cahors 1979, Société Préhistorique Française, p. 308-323, 2 fig.

SIMONNET R. (1996) - Approvisionnement en silex au Paléolithique supérieur : déplacements et caractéristiques physionomiques des paysages, l'exemple des Pyrénées centrales, in: Delporte H. et Clottes J. (dir.), Pyrénées Préhistoriques - Arts et Sociétés, actes du $118^{e}$ congrès national des sociétés historiques et scientifiques, Pau 1993, Éditions du C.T.H.S., p. 117-128, 10 fig.

SIMONNET R. (1998) - Le silex et la fin du Paléolithique supérieur dans le bassin de Tarascon-sur-Ariège, Préhistoire ariégeoise, Bulletin de la Suciété préhistorique Ariège-Pyrénées, t. LIII, p. 181-222, 6 fig., 4 tabl.

SMITH Ph. (1966) - Le solutréen en France, Bordeaux, Delmas, 449 p., 81 fig. (Public. Inst. Préhist. Univ. Bordeaux, 5).

\section{Pascal FOUCHER \\ Cristina SAN JUAN}

Service Régional de l'Archéologie

de Midi-Pyrénées

U.M.R. 5608 de Toulouse-Le-Mirail

7 , rue Chabanon, 31200 Toulouse 